\title{
Application of the cross wavelet transform and wavelet coherence to geophysical time series
}

\author{
A. Grinsted ${ }^{1}$, J. C. Moore ${ }^{1}$, and S. Jevrejeva ${ }^{2}$ \\ ${ }^{1}$ Arctic Centre, University of Lapland, Rovaniemi, Finland \\ ${ }^{2}$ Proudman Oceanographic Laboratory, Birkenhead, UK
}

Received: 11 June 2004 - Revised: 19 October 2004 - Accepted: 16 November 2004 - Published: 18 November 2004

Part of Special Issue "Nonlinear analysis of multivariate geoscientific data - advanced methods, theory and application"

\begin{abstract}
Many scientists have made use of the wavelet method in analyzing time series, often using popular free software. However, at present there are no similar easy to use wavelet packages for analyzing two time series together. We discuss the cross wavelet transform and wavelet coherence for examining relationships in time frequency space between two time series. We demonstrate how phase angle statistics can be used to gain confidence in causal relationships and test mechanistic models of physical relationships between the time series. As an example of typical data where such analyses have proven useful, we apply the methods to the Arctic Oscillation index and the Baltic maximum sea ice extent record. Monte Carlo methods are used to assess the statistical significance against red noise backgrounds. A software package has been developed that allows users to perform the cross wavelet transform and wavelet coherence (http://www.pol.ac.uk/home/research/waveletcoherence/).
\end{abstract}

\section{Introduction}

Geophysical time series are often generated by complex systems of which we know little. Predictable behavior in such systems, such as trends and periodicities, is therefore of great interest. Most traditional mathematical methods that examine periodicities in the frequency domain, such as Fourier analysis, have implicitly assumed that the underlying processes are stationary in time. However, wavelet transforms expand time series into time frequency space and can therefore find localized intermittent periodicities. There are two classes of wavelet transforms; the Continuous Wavelet Transform (CWT) and its discrete counterpart (DWT). The DWT is a compact representation of the data and is particularly useful for noise reduction and data compression whereas the CWT is better for feature extraction purposes.

Correspondence to: A. Grinsted

(ag@glaciology.net)
As we are interested in extracting low $\mathrm{s} / \mathrm{n}$ ratio signals in time series we discuss only CWT in this paper. While CWT is a common tool for analyzing localized intermittent oscillations in a time series, it is very often desirable to examine two time series together that may be expected to be linked in some way. In particular, to examine whether regions in time frequency space with large common power have a consistent phase relationship and therefore are suggestive of causality between the time series. Many geophysical time series are not Normally distributed and we suggest methods of applying the CWT to such time series. From two CWTs we construct the Cross Wavelet Transform (XWT) which will expose their common power and relative phase in time-frequency space. We will further define a measure of Wavelet Coherence (WTC) between two CWT, which can find significant coherence even though the common power is low, and show how confidence levels against red noise backgrounds are calculated.

We will present the basic CWT theory before we move on to XWT and WTC. New developments such as quantifying the phase relationship and calculating the WTC significance level will be treated more fully. When using the methods on time series it is important to have solid mechanistic foundations on which to base any relationships found, and we caution against using the methods in a "scatter-gun" approach (particularly if the time series probability density functions are modified). To illustrate how the various methods are used we apply them to two data sets from meteorology and glaciology. Finally, we will provide links to a MatLab software package.

\section{Data}

An example of two physical effects that we expect to be linked from consideration of the climate system are the mean winter state of the arctic atmosphere and winter severity reflected by ice conditions. 


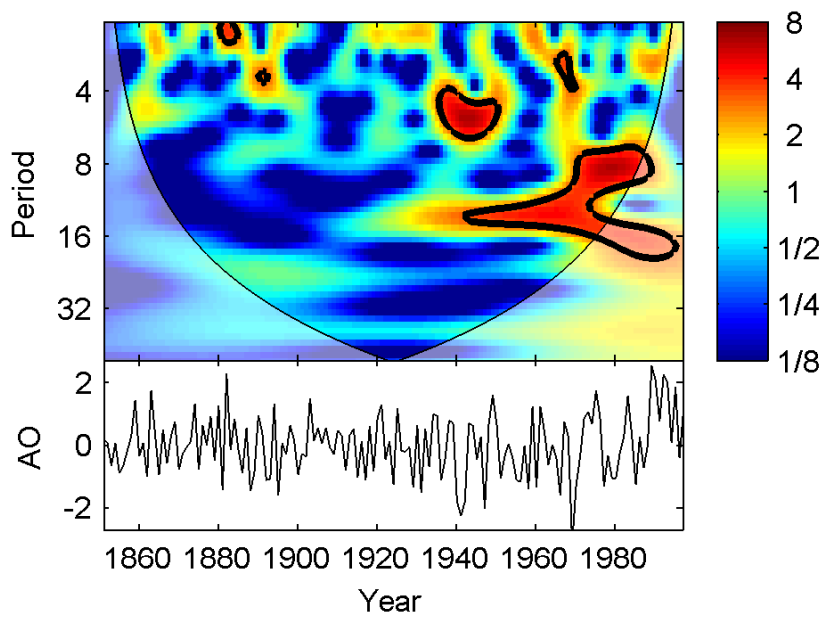

Fig. 1. The standardized time series of winter (DJF) AO (bottom) and its continuous wavelet power spectrum (top). The thick black contour designates the 5\% significance level against red noise and the cone of influence (COI) where edge effects might distort the picture is shown as a lighter shade. The standardized AO series has an AR1 coefficient of 0.02 .

The Arctic Oscillation (AO) is a key aspect of climate variability in the Northern Hemisphere. The AO is defined as the leading empirical orthogonal function (EOF) of Northern Hemisphere sea level pressure anomalies pole ward of $20^{\circ} \mathrm{N}$ (Thompson and Wallace, 1998), and characterized by an exchange of atmospheric mass between the Arctic and middle latitudes. The Baltic Sea is a transition zone between the North Atlantic region and the continental area of Eurasia, leading to large inter-annual variability of ice conditions. The Baltic Sea is partly covered by ice every winter season, the maximum annual ice extent varies between $10-100 \%$ of the sea area, the length of ice season is 4-7 months, and the maximum annual thickness of ice is $50-120 \mathrm{~cm}$ (Jevrejeva, 2001; Seinä et al., 2001). Recently published results demonstrate that large-scale atmospheric circulation patterns in the Arctic and North Atlantic described by the AO or by the somewhat similar North Atlantic Oscillation teleconnections significantly control ice conditions in the Baltic Sea (Loewe and Koslowski, 1998; Omstedt and Chen, 2001; Jevrejeva and Moore, 2001; Jevrejeva, 2002).

In this paper, we examine the connection between winter AO and Baltic Sea ice extent and especially explore the phase relationships between the series in the light of the expected causality links. Ice conditions are represented by the time series of maximum annual ice extent in the Baltic Sea (BMI) for the period 1720-2000 (Seinä et al., 2001). We use the winter AO index (December-February 1851-1997) of Thompson and Wallace (1998).

Many statistical tests assume that the probability density function (pdf) is close to Normal. Our experience with CWTs of geophysical time series shows that series far from normally distributed produces rather unreliable and less significant results. Occasionally it can therefore be a good idea

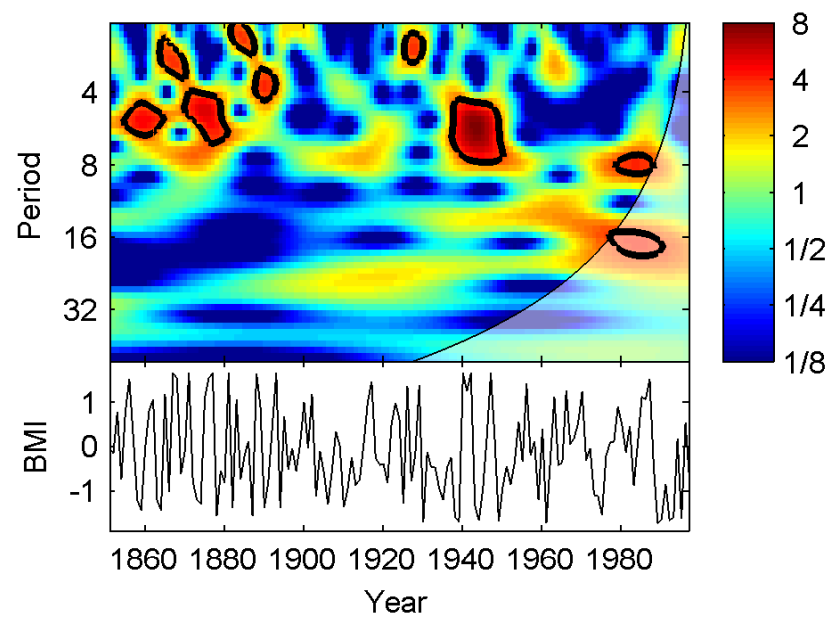

Fig. 2. The standardized BMI percentile time series (bottom) and its continuous wavelet power (top). The thick contour designates the $5 \%$ significance level against red noise and the cone of influence (COI) where edge effects might distort the picture is shown as a lighter shade. The standardized BMI percentile series has an AR1 coefficient of 0.08 .

to transform the pdf of the time series. However, we caution against rashly changing the pdf. The BMI index is bi-modally distributed with maximum probabilities around $70000 \mathrm{~km}^{2}$ and $420000 \mathrm{~km}^{2}$. A simple periodic oscillation between these two modes will almost have the shape of a square wave. The power of a square wave leaks into frequency bands outside the fundamental period. We therefore transform the BMI index into a record of percentiles (in terms of its cumulative distribution function) and thus forcing the pdf to be rectangular. This has the effect of narrowing the bandwidth of intermittent oscillations. We standardize (zero mean, unit standard deviation) both time series and will refer to the standardized versions as simply AO and BMI. The time series of the $\mathrm{AO}$ and $\mathrm{BMI}$ time series are shown in Figs. 1 and 2.

\section{Methods}

\subsection{The Continuous Wavelet Transform (CWT)}

A wavelet is a function with zero mean and that is localized in both frequency and time. We can characterize a wavelet by how localized it is in time $(\Delta t)$ and frequency $(\Delta \omega$ or the bandwidth). The classical version of the Heisenberg uncertainty principle tells us that there is always a tradeoff between localization in time and frequency. Without properly defining $\Delta t$ and $\Delta \omega$, we will note that there is a limit to how small the uncertainty product $\Delta t \cdot \Delta \omega$ can be. One particular wavelet, the Morlet, is defined as

$\psi_{0}(\eta)=\pi^{-1 / 4} e^{i \omega_{0} \eta} e^{-\frac{1}{2} \eta^{2}}$.

where $\omega_{0}$ is dimensionless frequency and $\eta$ is dimensionless time. When using wavelets for feature extraction purposes 
the Morlet wavelet (with $\omega_{0}=6$ ) is a good choice, since it provides a good balance between time and frequency localization. We therefore restrict our further treatment to this wavelet, although the methods we present are generally applicable (see, e.g. Foufoula-Georgiou, 1995).

The idea behind the CWT is to apply the wavelet as a bandpass filter to the time series. The wavelet is stretched in time by varying its scale $(s)$, so that $\eta=s \cdot t$, and normalizing it to have unit energy. For the Morlet wavelet (with $\omega_{0}=6$ ) the Fourier period $\left(\lambda_{w t}\right)$ is almost equal to the scale $\left(\lambda_{w t}=1.03 \mathrm{~s}\right)$. The CWT of a time series $\left(x_{n}, n=1, \ldots, N\right)$ with uniform time steps $\delta t$, is defined as the convolution of $x_{n}$ with the scaled and normalized wavelet. We write

$W_{n}^{X}(s)=\sqrt{\frac{\delta t}{s}} \sum_{n^{\prime}=1}^{N} x_{n^{\prime}} \psi_{0}\left[\left(n^{\prime}-n\right) \frac{\delta t}{s}\right]$.

In practice it is faster to implement the convolution in Fourier space (see details in Torrence and Compo, 1998). We define the wavelet power as $\left|W_{n}^{X}(s)\right|^{2}$. The complex argument of $W_{n}^{X}(s)$ can be interpreted as the local phase.

The CWT has edge artifacts because the wavelet is not completely localized in time. It is therefore useful to introduce a Cone of Influence (COI) in which edge effects can not be ignored. Here we take the COI as the area in which the wavelet power caused by a discontinuity at the edge has dropped to $\mathrm{e}^{-2}$ of the value at the edge.

The statistical significance of wavelet power can be assessed relative to the null hypotheses that the signal is generated by a stationary process with a given background power spectrum $\left(P_{k}\right)$. Many geophysical time series have distinctive red noise characteristics that can be modeled very well by a first order autoregressive (AR1) process. The Fourier power spectrum of an AR1 process with lag-1 autocorrelation $\alpha$ (estimated from the observed time series e.g. Allen and Smith, 1996) is given by

$$
P_{k}=\frac{1-\alpha^{2}}{\left|1-\alpha e^{-2 i \pi k}\right|^{2}}
$$

where $\mathrm{k}$ is the Fourier frequency index.

The wavelet transform can be thought of as a consecutive series of band-pass filters applied to the time series where the wavelet scale is linearly related to the characteristic period of the filter $\left(\lambda_{w t}\right)$. Hence, for a stationary process with the power spectrum $P_{k}$ the variance at a given wavelet scale, by invocation of the Fourier convolution theorem, is simply the variance in the corresponding band of $P_{k}$. If $P_{k}$ is sufficiently smooth then we can approximate the variance at a given scale simply with $P_{k}$ using the conversion $k^{-1}=\lambda_{w t}$. Torrence and Compo (1998) use Monte Carlo methods to show that this approximation is very good for the AR1 spectrum. They then show that the probability that the wavelet power, of a process with a given power spectrum $\left(P_{k}\right)$, being greater than $p$ is

$D\left(\frac{\left|W_{n}^{X}(s)\right|^{2}}{\sigma_{X}^{2}}<p\right)=\frac{1}{2} P_{k} \chi_{v}^{2}(p)$

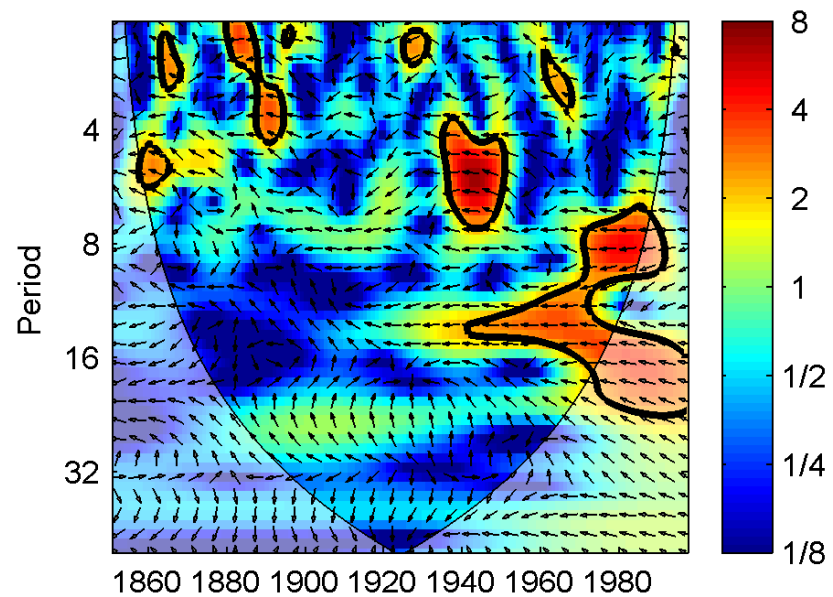

Fig. 3. Cross wavelet transform of the standardized AO and BMI time series. The 5\% significance level against red noise is shown as a thick contour. The relative phase relationship is shown as arrows (with in-phase pointing right, anti-phase pointing left, and BMI leading $\mathrm{AO}$ by $90^{\circ}$ pointing straight down).

where $v$ is equal to 1 for real and 2 for complex wavelets.

The CWT of the AO and BMI are shown in Figs. 1 and 2 respectively. There are clearly common features in the wavelet power of the two time series such as the significant peak in the $\sim 5$ year band around 1940. Both series also have high power in the 2-7 year band in the period from 1860-1900, though for AO the power is not above the 5\% significance level. However, the similarity between the portrayed patterns in this period is quite low and it is therefore hard to tell if it is merely a coincidence. The cross wavelet transform helps in this regard.

\subsection{The cross wavelet transform}

The cross wavelet transform (XWT) of two time series $x_{n}$ and $y_{n}$ is defined as $W^{X Y}=W^{X} W^{Y *}$, where * denotes complex conjugation. We further define the cross wavelet power as $\left|W^{X Y}\right|$. The complex argument $\arg \left(W^{x y}\right)$ can be interpreted as the local relative phase between $x_{n}$ and $y_{n}$ in time frequency space. The theoretical distribution of the cross wavelet power of two time series with background power spectra $P_{k}^{X}$ and $P_{k}^{Y}$ is given in Torrence and Compo (1998) as

$D\left(\frac{\left|W_{n}^{X}(s) W_{n}^{Y *}(s)\right|}{\sigma_{X} \sigma_{Y}}<p\right)=\frac{Z_{v}(p)}{v} \sqrt{P_{k}^{X} P_{k}^{Y}}$,

where $Z_{v}(p)$ is the confidence level associated with the probability $p$ for a pdf defined by the square root of the product of two $\chi^{2}$ distributions. For example the 5\% significance level marked in Fig. 3 is calculated using $Z_{2}(95 \%)=3.999$.

The XWT of AO and BMI is shown in Fig. 4. Here we notice that the common features we found by eye from the individual wavelet transforms stand out as being significant at the 5\% level. We also note that there also is significant common power in the $\sim 10-16$ year band from 1940-1980. 


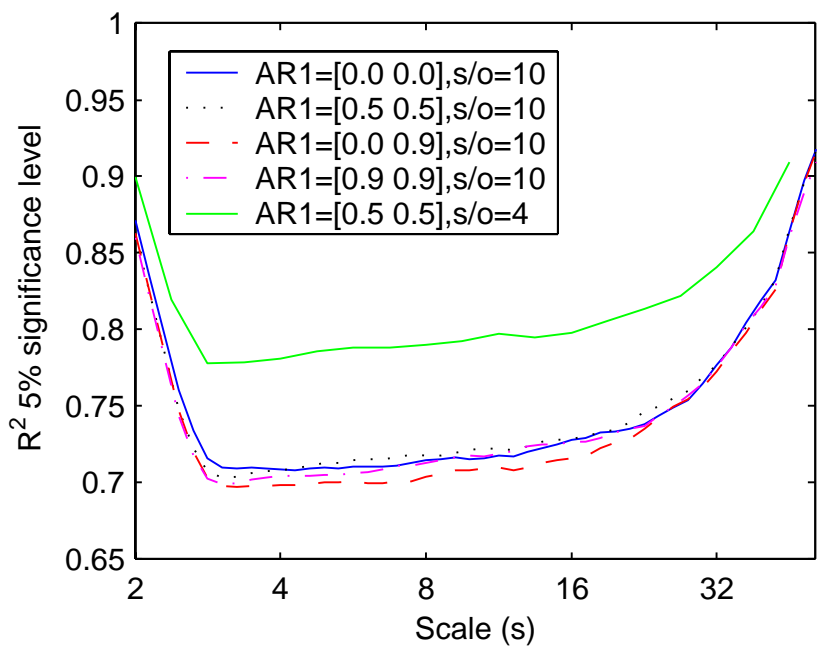

Fig. 4. Wavelet coherence 5\% significance level determined using Monte Carlo generated noise (with 10000 surrogate data set pairs). The legend shows the 2 AR 1 coefficients of the surrogate data sets and the numbers of scales per octave (s/o) used in calculating the scale smoothing. The color of the noise has little impact on the significance level, whereas the specifics of the smoothing have a large impact. The large values at either end of the spectrum are due to the scale smoothing operator reaching the scale boundaries.

For there to be a simple cause and effect relationship between the phenomena recorded in the time series we would expect that the oscillations are phase locked. So, it is comforting that the XWT show that AO and BMI are in anti phase in all the sectors with significant common power. Since AO and BMI are in anti-phase across all scales, we conclude that BMI to a large extent simply mirrors the AO. Outside the areas with significant power the phase relationship is also predominantly anti phase. We therefore speculate that there is a stronger link between $\mathrm{AO}$ and $\mathrm{BMI}$ than that implied by the cross wavelet power.

\subsection{Cross wavelet phase angle}

As we are interested in the phase difference between the components of the two time series we need to estimate the mean and confidence interval of the phase difference. We use the circular mean of the phase over regions with higher than 5\% statistical significance that are outside the COI to quantify the phase relationship. This is a useful and general method for calculating the mean phase. The circular mean of a set of angles $\left(a_{i}, i=1 \ldots n\right)$ is defined as (e.g. Zar, 1999)

$a_{m}=\arg (X, Y)$ with $X=\sum_{i=1}^{n} \cos \left(a_{i}\right)$ and $Y=\sum_{i=1}^{n} \sin \left(a_{i}\right)$,

It is difficult to calculate the confidence interval of the mean angle reliably since the phase angles are not independent. The number of angles used in the calculation can be set arbitrarily high simply by increasing the scale resolution. However, it is interesting to know the scatter of angles around the mean. For this we define the circular standard deviation as

$s=\sqrt{-2 \ln (R / n)}$,

where $R=\sqrt{ }\left(X^{2}+Y^{2}\right)$. The circular standard deviation is analogous to the linear standard deviation in that it varies from zero to infinity. It gives similar results to the linear standard deviation when the angles are distributed closely around the mean angle. In some cases there might be reasons for calculating the mean phase angle for each scale, and then the phase angle can be quantified as a number of years.

The XWT phase angle within the $5 \%$ significant regions and outside the COI has the mean phase $-176 \pm 12^{\circ}$ (where \pm designates the circular standard deviation). This basically confirms the conclusion that $\mathrm{AO}$ and BMI are in anti-phase. Note that the time series already have a 3 month lag since the ice extent is greatest in April. A 3 month lag is consistent with the mechanism of stratospheric forcing of the troposphere (Baldwin et al., 2001; Jevrejeva et al., 2003). The observation that the phase angles are constant across all scales argues for a constant time lag due to the physical mechanism of signal propagation from the AO to the ice extent. The deviation from completely anti-phase suggests that $A O$ leads BMI slightly, however, the circular standard deviation is too large to make any firm conclusion.

\subsection{Wavelet coherence}

Cross wavelet power reveals areas with high common power. Another useful measure is how coherent the cross wavelet transform is in time frequency space. Following Torrence and Webster (1998) we define the wavelet coherence of two time series as

$$
R_{n}^{2}(s)=\frac{\left|S\left(s^{-1} W_{n}^{X Y}(s)\right)\right|^{2}}{S\left(s^{-1}\left|W_{n}^{X}(s)\right|^{2}\right) \cdot S\left(s^{-1}\left|W_{n}^{Y}(s)\right|^{2}\right)},
$$

where $S$ is a smoothing operator. Notice that this definition closely resembles that of a traditional correlation coefficient, and it is useful to think of the wavelet coherence as a localized correlation coefficient in time frequency space. We write the smoothing operator $S$ as

$S(W)=S_{\text {scale }}\left(S_{\text {time }}\left(W_{n}(s)\right)\right)$,

where $S_{\text {scale }}$ denotes smoothing along the wavelet scale axis and $S_{\text {time }}$ smoothing in time. It is natural to design the smoothing operator so that it has a similar footprint as the wavelet used. For the Morlet wavelet a suitable smoothing operator is given by Torrence and Webster (1998)

$$
\begin{gathered}
\left.S_{\text {time }}(W)\right|_{s}=\left.\left(W_{n}(s) * c_{1}^{\frac{-t^{2}}{2 s^{2}}}\right)\right|_{s}, \\
\left.S_{\text {time }}(W)\right|_{s}=\left.\left(W_{n}(s) * c_{2} \Pi(0.6 \mathrm{~s})\right)\right|_{n},
\end{gathered}
$$

where $c_{1}$ and $c_{2}$ are normalization constants and $\Pi$ is the rectangle function. The factor of 0.6 is the empirically determined scale decorrelation length for the Morlet wavelet (Torrence and Compo, 1998). In practice both convolutions are 
done discretely and therefore the normalization coefficients are determined numerically.

The statistical significance level of the wavelet coherence is estimated using Monte Carlo methods. We generate a large ensemble of surrogate data set pairs with the same AR1 coefficients as the input datasets. For each pair we calculate the wavelet coherence. We then estimate the significance level for each scale using only values outside the COI. Empirical testing shows that the AR1 coefficients have little impact on the significance level. The specifics of the smoothing operator, however, have a large impact. For example the resolution chosen when calculating the scale smoothing has a major influence on the significance level (see Fig. 4). The Monte Carlo estimation of the significance level requires of the order of 1000 surrogate data set pairs. The number of scales per octave should be high enough to capture the rectangle shape of the scale smoothing operator while minimizing computing time. Empirically we find 10 scales per octave to be satisfactory.

The squared WTC of AO and BMI is shown in Fig. 5. Compared with the XWT a larger section stands out as being significant and all these areas show an anti-phase relationship between $\mathrm{AO}$ and BMI. The area of a time frequency plot above the 5\% significance level is not a reliable indication of causality. Even if the scales were appropriately weighed for the averaging, it is possible for two series to be perfectly correlated at one specific scale while the area of significant correlation is much less than 5\%. However, the significant region of Fig. 5 is so extensive that it is very unlikely that this is simply by chance. Oscillations in $\mathrm{AO}$ are manifested in the BMI on wavelengths varying from 2-20 years, suggesting that BMI passively mirrors AO. Regions with low coherence coincide with low wavelet power in the $\mathrm{AO}$ and are therefore expected. Possibly because the EOF that AO represents is not really capturing the actual location of the centers of action during the Little Ice Age. As with the XWT, we can calculate the mean phase angle of the significant regions. The mean phase angle over the regions with significant wavelet coherence and outside the COI is $174 \pm 15^{\circ}$.

\section{Summary}

The CWT expands a time series into a time frequency space where oscillations can be seen in a highly intuitive way. The Morlet wavelet (with $\omega_{0}=6$ ) is a good choice when using wavelets for feature extraction purposes, because it is reasonably localized in both time and frequency. From the CWTs of two time series one can construct the XWT. The XWT exposes regions with high common power and further reveals information about the phase relationship. If the two series are physically related we would expect a consistent or slowly varying phase lag that can be tested against mechanistic models of the physical process. WTC can be thought of as the local correlation between two CWTs. In this way locally phase locked behavior is uncovered. The more desirable features of the WTC come at the price of being slightly less localized

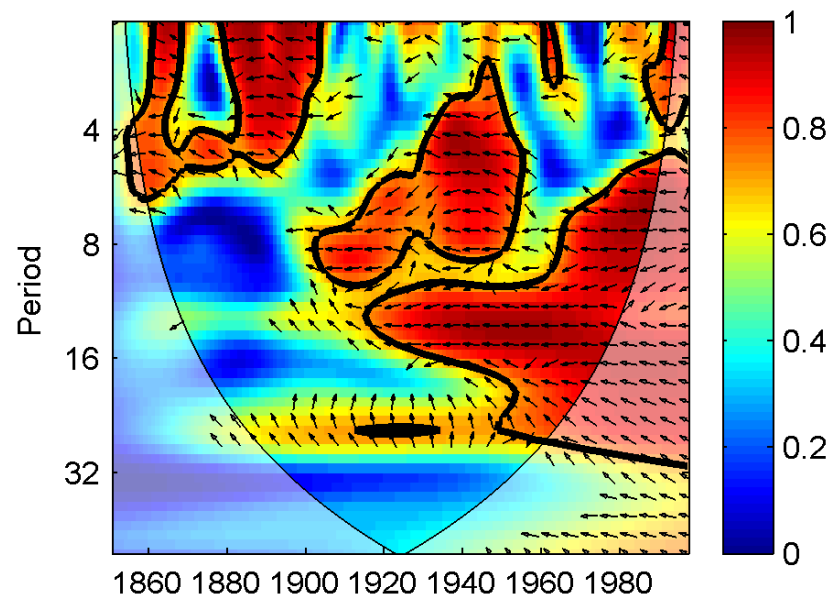

Fig. 5. Squared wavelet coherence between the standardized AO and BMI time series. The 5\% significance level against red noise is shown as a thick contour. All significant sections show anti-phase behavior.

in time frequency space. The significance level of the WTC has to be determined using Monte Carlo methods.

\subsection{Practical tips}

Cross wavelet analysis and wavelet coherence are powerful methods for testing proposed linkages between two time series.

- Check the histograms of the time series to ensure that they are not too far from normally distributed. Consider transforming the time series, if the pdf's of the time series are far from Gaussian. When choosing a transformation, it is preferable to choose an analytic transformation such as taking the logarithm if the data is lognormally distributed. In other cases the simple "percentile" transformation we used for the BMI might be useful. An advantage of using that particular transformation is that it does not have any outliers.

- Consider what the expectations are for the outcome of the analysis given the proposed linking mechanism. We caution against blindly applying these methods to randomly chosen data sets. Like other statistical tests some data set sets will display highly statistically significant links simply by chance.

- When a wavelet has been chosen the CWTs of both time series are calculated. We suggest a scale resolution of 10 scales per octave and use of the Morlet wavelet unless there are good grounds to do otherwise. For geophysical time series an AR1 red noise assumption is often suitable and Eqs. (3) and (4) can be used to calculate the significance level of the wavelet power. Remember to take special care not to misinterpret results inside the COI. 
- From the two CWTs the XWT is calculated. The XWT exposes regions with high common power and further reveals information about the phase relationship. If the two series are physically related we would expect a consistent or slowly varying phase lag that can be tested against mechanistic models of the physical process. The circular mean of the phase angles can be used to quantify the phase relationship.

- Also, from two CWTs the WTC can be calculated which can be thought of as the local correlation between the time series in time frequency space. Where XWT unveils high common power, WTC finds locally phase locked behavior. The more desirable features of the WTC come at the price of being slightly less localized in time frequency space. The significance level of the WTC has to be determined using Monte Carlo methods.

A MatLab software package by the authors for performing XWT and WTC can be found at http://www.pol.ac.uk/home/ research/waveletcoherence/.

Acknowledgements. Financial assistance was provided by the Thule Institute. Some of our software includes code originally written by $\mathrm{C}$. Torrence and G. Compo that is available at: http://paos.colorado.edu/research/wavelets/ and by E. Breitenberger of the University of Alaska which were adapted from the freeware SSA-MTM Toolkit: http://www.atmos.ucla.edu/tcd/ssa.

Edited by: M. Thiel

Reviewed by: two referees

\section{References}

Allen, M. R. and Smith, L. A.: Monte Carlo SSA: Detecting irregular oscillations in the presence of coloured noise, J. Clim., 9, 3373-3404, 1996.

Baldwin, M. P., Gray, L. J., Dunkerton, T. J., Hamilton, K., Haynes, P. H., Randel, W. J., Holton, J. R., Alexander, M. J., Hirota, I., Horinouchi, T., Jones, D. B. A., Kinnerslay, J. S., Marquardt, C., Sato, K., and Tarahashi, M.: The Quasi-Biennial Oscillation, Rev. Geophys., 39, 170-229, 2001.

Foufoula-Georgiou, E. and Kumar, P.: Wavelets in Geophysics, Academic Press, 373, 1995.

Jevrejeva, S. and Moore, J. C.: Singular Spectrum Analysis of Baltic Sea ice conditions and large-scale atmospheric patterns since 1708, Geophys. Res. Lett., 28, 4503-4507, 2001.

Jevrejeva, S.: Association between the ice conditions in the Baltic Sea and the North Atlantic Oscillation, Nordic Hydrol., 33, 319330, 2002.

Jevrejeva, S., Moore, J. C., and Grinsted, A.: Influence of the Arctic Oscillation and El Ninõ-Southern Oscillation (ENSO) on ice conditions in the Baltic Sea: The wavelet approach, J. Geophys. Res., 108(D21), 4677, doi:10.1029/2003JD003417, 2003.

Loewe, P. and Koslowski, G. : The Western Baltic Sea ice season in terms of a mass-related severity index 1879-1992, II Spectral characteristics and associations with NAO, QBO and solar cycle, Tellus, 50A, 219-241, 1998.

Omstedt, A. and Chen, D.: Influence of atmospheric circulation on the maximum ice extent in the Baltic Sea, J. Geophys. Res., Vol. 106, 4493-4500, 2001.

Seinä, A., Grönvall, H., Kalliosaari, S., and Vainio, J.: Ice seasons 1996-2000 in Finnish sea areas / Jäätalvet 1996-2000 Suomen merialueilla, Meri, Report Series of the Finnish Institute of Marine Research, 43, 2001.

Thompson, D. W. J. and Wallace, J. M.: The Arctic Oscillation signature in the winter geopotential height and temperature fields, Geophys. Res. Lett., 25, 1297-1300, 1998.

Torrence, C. and Compo, G. P.: A practical guide to wavelet analysis, Bull. Am. Meteorol. Soc., 79, 61-78, 1998.

Torrence, C. and Webster, P.: Interdecadal Changes in the ESNOMonsoon System, J. Clim., 12, 2679-2690, 1999.

Zar, J. H.: Biostatistical Analysis, Prentice hall, New Jersey, 1999. 